\title{
Medium size telescopes for the Cherenkov Telescope Array
}

\section{Markus Garczarczyk}

Markus Garczarczyk, "Medium size telescopes for the Cherenkov Telescope Array," Proc. SPIE 10700, Ground-based and Airborne Telescopes VII, 1070023 (6 July 2018); doi: $10.1117 / 12.2312000$

Event: SPIE Astronomical Telescopes + Instrumentation, 2018, Austin, Texas, United States 


\title{
Medium Size Telescopes for the Cherenkov Telescope Array
}

\author{
Markus Garczarczyk ${ }^{\mathrm{a}}$ and the CTA Consortium ${ }^{\mathrm{b}}$ \\ ${ }^{a}$ Deutsches Elektronen Synchrotron (DESY), Platanenallee 6, D-15738 Zeuthen, Germany \\ ${ }^{b}$ see http://www.cta-observatory.com for full author and affiliation list
}

\begin{abstract}
The Cherenkov Telescope Array (CTA) is the next generation ground-based observatory for gamma-ray astronomy at very high energies in the range from $20 \mathrm{GeV}$ to $300 \mathrm{TeV} .{ }^{1}$ In order to cover the entire sky an observatory with two telescope arrays is planned, one in the southern hemisphere and one in the northern hemisphere. Each site will combine imaging air Cherenkov telescopes of different sizes and designs to cover the very wide energy range. These sites will complement each other, providing full-sky coverage for galactic and extra-galactic sources. At least three telescope types are required to cover the full CTA energy range in a cost-effective way. The sensitivity in the core energy range between $150 \mathrm{GeV}$ and $5 \mathrm{TeV}$ will be dominated by up to 40 Medium Size Telescopes (MSTs) distributed over both observatory sites. It is intended to equip the MSTs with FlashCam and NectarCAM cameras. This document describes the aspects of the MST design and the status of commissioning and performance validation of the individual assemblies.
\end{abstract}

Keywords: CTA, MST, FlashCam, NectarCAM

\section{INTRODUCTION}

There are two proposed telescope designs for the MST: A modified Davies-Cotton and a Schwarzschild-Couder design (SCT). ${ }^{2}$ Here we focus on the modified Davies-Cotton MST design, which is a telescope with a reflector diameter of $12 \mathrm{~m}$, focal length of $16 \mathrm{~m}$ mounted on a Alt-Az mount. Figure 1 shows the schematics of the telescope and indicates the main telescope assemblies.

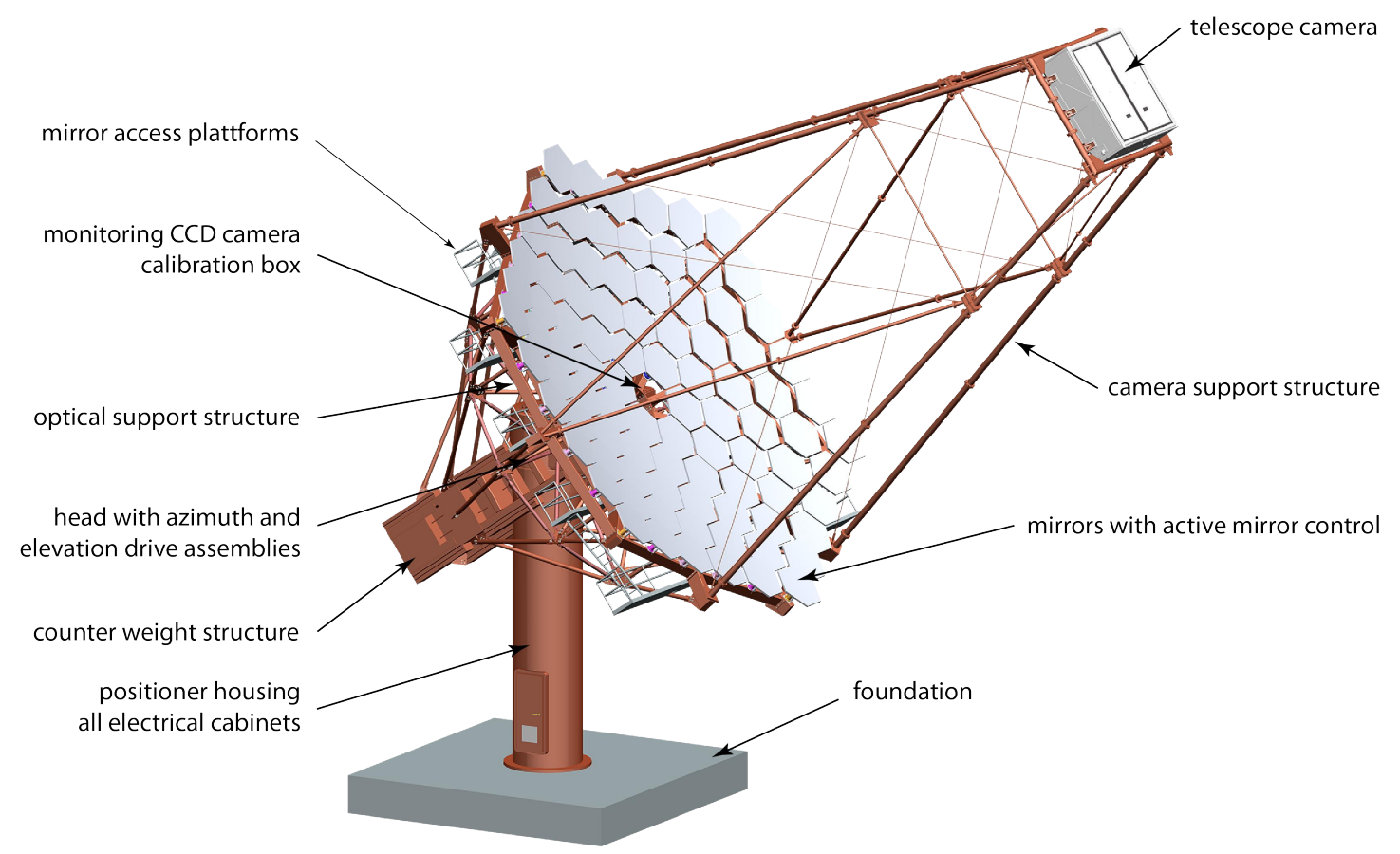

Figure 1. Model of the MST. The camera maintenance structure with its foundation and auxiliary cabinets planned to be installed on the telescope foundation are not shown in this view.

Ground-based and Airborne Telescopes VII, edited by Heather K. Marshall,

Jason Spyromilio, Proc. of SPIE Vol. 10700, 1070023 • @ 2018 SPIE

CCC code: $0277-786 \mathrm{X} / 18 / \$ 18 \cdot$ doi: $10.1117 / 12.2312000$ 
The MST project is organized around three sub-projects: The MST structure, the FlashCam and the NectarCAM. Currently, 8 countries with 27 institutes and of the order of 125 persons are involved in these three projects. The baseline of CTA is to deploy 25 MSTs at the Southern site (foreseen to be hosted by ESO close to the Paranal site in Chile) and 15 MSTs at the Northern site, hosted by IAC at La Palma (Canary Islands, Spain). In the initial stage of the CTA project, for which funding is currently being secured, 15 MSTs will be deployed in Chile and 5 MSTs in La Palma. Following the progress of the observatory infrastructure first MSTs are expected to be deployed in the year 2020 .

\section{THE STRUCTURE OF THE MEDIUM SIZE TELESCOPE}

The required pointing precision of 7", ability to point to any coordinates on the sky within $90 \mathrm{~s}$, optical point spread function (PSF) smaller than the Cherenkov camera's pixel size and an effective mirror area larger than $88 \mathrm{~m}^{2}$ are the key parameters used for the design of the telescope structure. ${ }^{3}$ The telescope is made out of steel to ensure sufficient stiffness of the optical support structure under varying elevation angles, without the need of active mirror alignment during the observation. The total weight of the telescope is $86 \mathrm{t}$.

The telescope optics is arranged as a modified Davies-Cotton design. This is a tradeoff to optimise the PSF over a large fraction of the Cherenkov camera's field of view (FoV) and to improve the isochronocity of the reflector. The reflector is tessellated and consists of 86 hexagonally-shaped mirrors with $1.2 \mathrm{~m}$ flat-to-flat side length. The mirrors are mounted on a sphere of $19.2 \mathrm{~m}$ radius. The distance between the imaginary central mirror and the focal plane of the camera is $16.0 \mathrm{~m}$, while the spherical mirror segments themselves have a radius of curvature of

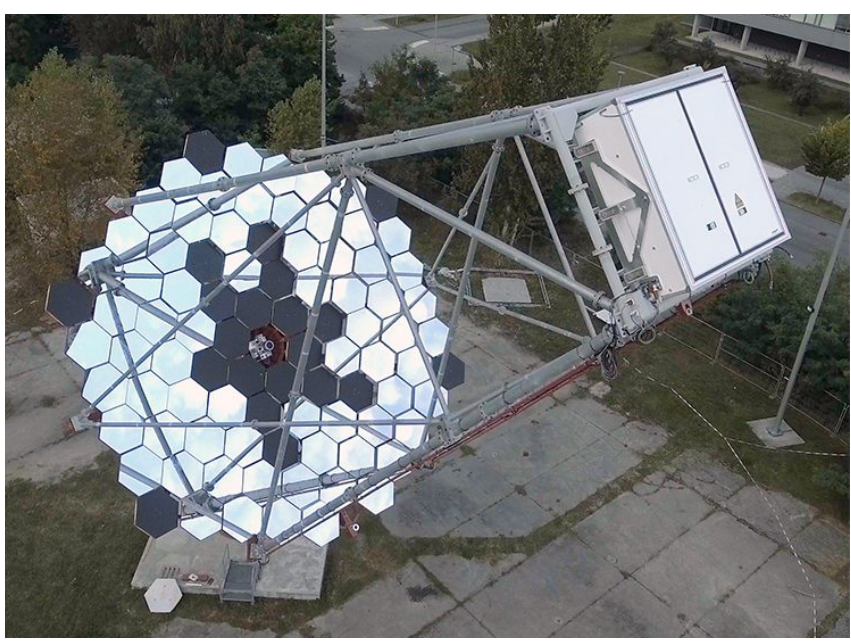

Figure 2. The MST prototype with the FlashCam Cherenkov camera during the integration test in Berlin-Adlershof. Due to limited number of actuators the central and outermost mirrors are filled with black painted dummies. Image taken in September 2017. $32.14 \mathrm{~m}$, i.e. approximately twice the focal length, and are aligned to reflect rays parallel to the optical axis into the focal point.

Several mirror designs are proposed for the MST, which are all based on cold slumping technology. This reduces the costs and allows reproducible optical performance during mass production, when comparing with solid glass mirrors. The mirror spherical shape is produced by bending of a thin glass sheet on a precisely formed mould. Reinforcement of the mirror is made using an aluminium honeycomb sandwich panel glued to the glass. A second thin glass sheet on the rear side of the mirror together with side walls are closing the sandwich and making it water tight. Cherenkov telescopes are typically not protected by a dome. The mirror coating needs to withstand the environmental impacts, e.g. humidity/rain and sand abrasion. The coating is made of a reflective aluminium layer, protected by a multilayer of $\mathrm{SiO}_{2}$ and $\mathrm{HfO}_{2}$ or $\mathrm{ZrO}_{2}$.

Each mirror is held by two motor driven actuators and a fix point to a mirror support unit, which itself is screwed on the dish. The actuators are used to align the individual mirrors to form a homogenous reflector during the initial telescope assembly. Mirror access platforms on the back of the dish structure allow easy access to the components during maintenance. The positioner of the telescope is designed as a cylindrical tower with $2 \mathrm{~m}$ diameter and $9 \mathrm{~m}$ height. The tower is separated by three floors and hosts electrical cabinets, the azimuth drive and the lubrication assembly. The elevation drive is mounted on the two sides of the head, outside of the positioner.

Accurate calibration of telescope pointing requires precise knowledge of the true pointing direction, as well as the input (Alt, Az) drive assembly coordinates and the target pointing direction. The MST pointing calibration makes use of a single, wide FoV CCD camera (SingleCCD) installed in the dish centre, aligned to the optical 
axis of the telescope and facing the Cherenkov camera. ${ }^{4}$ The FoV of this camera is chosen sufficiently large to observe both the star field (to determine the direction of the optical axis of the SingleCCD) and the pointing LEDs mounted on the Cherenkov camera body (to determine its orientation w.r.t. the optical axis).

A prototype of the MST structure is shown in Figure 2. It has been built in Berlin-Adlershof and has been used extensively over the last years to gain experience with the design and develop improvements focused mainly on cost reduction and mass production capabilities. The prototype has also been used to develop and debug the telescope and array control software. ${ }^{5-7}$ The design optimisations incorporated in the last years profit from the early construction of the prototype telescope as well as close cooperation with the single mirror small size telescope $(\mathrm{SST}-1 \mathrm{M})^{8}$ and the production of the positioner for the SCT. The following design changes can be highlighted:

- The pointing accuracy of the azimuth axis has been improved by relocating the shaft encoder and implementing an dedicated, denser teeth encoder ring at the azimuth bearing.

- The weight and shadowing of the mirrors by the camera support structure was reduced by using thinner tubes and replacing some of them by pre-tensioned steel wires.

- The stiffness of the OSS, its weight as well as production and assembly effort was improved by redesigning the dish. The new dish has straight beams interconnected with each other in a way that direct support for each mirror segment is given.

- Long term temperature cycling and exposure to the environment at the prototype telescope helped to identify possible problems in the design, material selection and production procedures of the mirrors.

- The housing of the SingleCCD camera was modified to reduce reflections on the glass window and inner side walls. Heating of the front window and temperature / humidity sensors were incorporated.

The prototype telescope is operated almost every night remotely. The main tasks are focused on performance evaluation and commissioning, as well as software development of the individual telescope assemblies. Major achievements in the past months are the automatic routines for mirror alignment using an artificial light source as well as stars ${ }^{9}$ and the improvement of the tracking accuracy by implementing a bending model to compensate for the telescope structure imperfections. The progress is significantly affected by the bright night sky background and limited number of clear nights in the area of Berlin.

\section{MST CHERENKOV CAMERAS}

Two types of Cherenkov cameras are proposed for the MSTs. While the two designs are based on fundamentally different digitization and trigger concepts, and on different architectures, both use high quantum-efficiency photomultipliers (PMTs) to record the incoming Cherenkov light of the air-shower events. The trigger and readout electronics are located inside the camera housings. The cameras have a FoV of above $7.5^{\circ}$ diameter. The $>1750$ pixels are arranged in a hexagonal matrix of $50 \mathrm{~mm}$ spacing, corresponding to $0.18^{\circ}$ pixel size in the telescope focal plane. The cameras are mechanically supported at the primary focus of the telescope reflector by the camera support structure, and are interfaced by power, liquid cooling and dry air pipes, as well as by optical fibre cables for control and bulk data transfer. These interfaces are designed such that both camera types fit into the telescope. The design and status of the two camera sub-projects are described in more detail in the following two sections.

\subsection{FlashCam}

The FlashCam camera features as one of their core elements a fully digital trigger and readout system that has been designed, prototyped and successfully tested over the past years. Figure 3 shows the FlashCam installed for integration tests at the MST prototype in Berlin-Adlershof, when also first Cherenkov images of air showers have been recorded with the MST. Cherenkov telescope cameras need to create an event trigger from the incoming light distribution by themselves. Short timescales are necessary to minimize dead-time. In FlashCam, PMT signals 

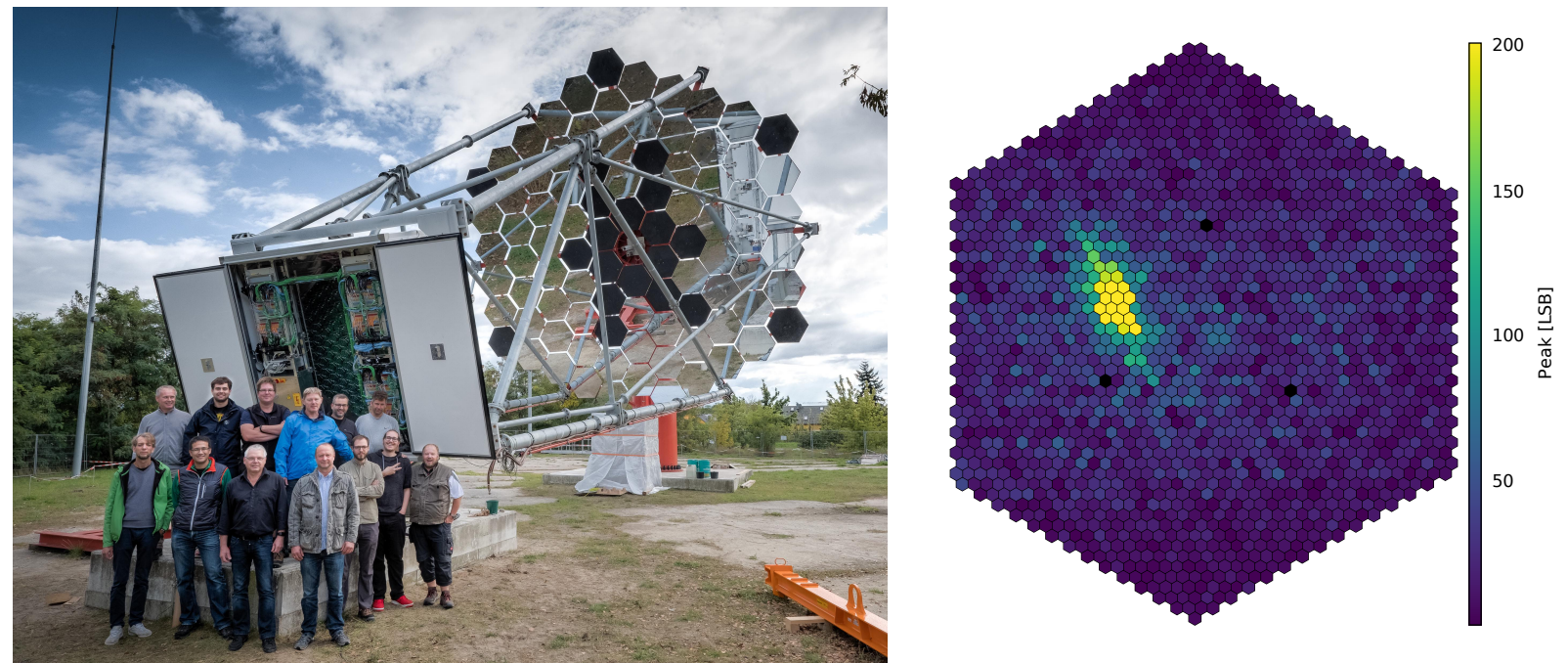

Figure 3. The FlashCam mounted at the MST structure prototype for integration tests, with one of the first cosmic air shower events recorded by the telescope. Image taken in September 2017.

are digitized, after pre-amplification and shaping, using commercial low-power $250 \mathrm{MHz}$ pipeline-ADCs. The trigger creation, as well as further pre-processing of the digitized signals is performed in the front-end electronics by commercial low-cost FPGA. The design, including a preamplifier that behaves deterministically non-linearly at high incoming charges, permits to digitize the full dynamic range of a pixel $(\sim 0.2-3000$ photo-electrons [p.e.]) in one channel, saving costs as well as bandwidth of the subsequent data transfer.

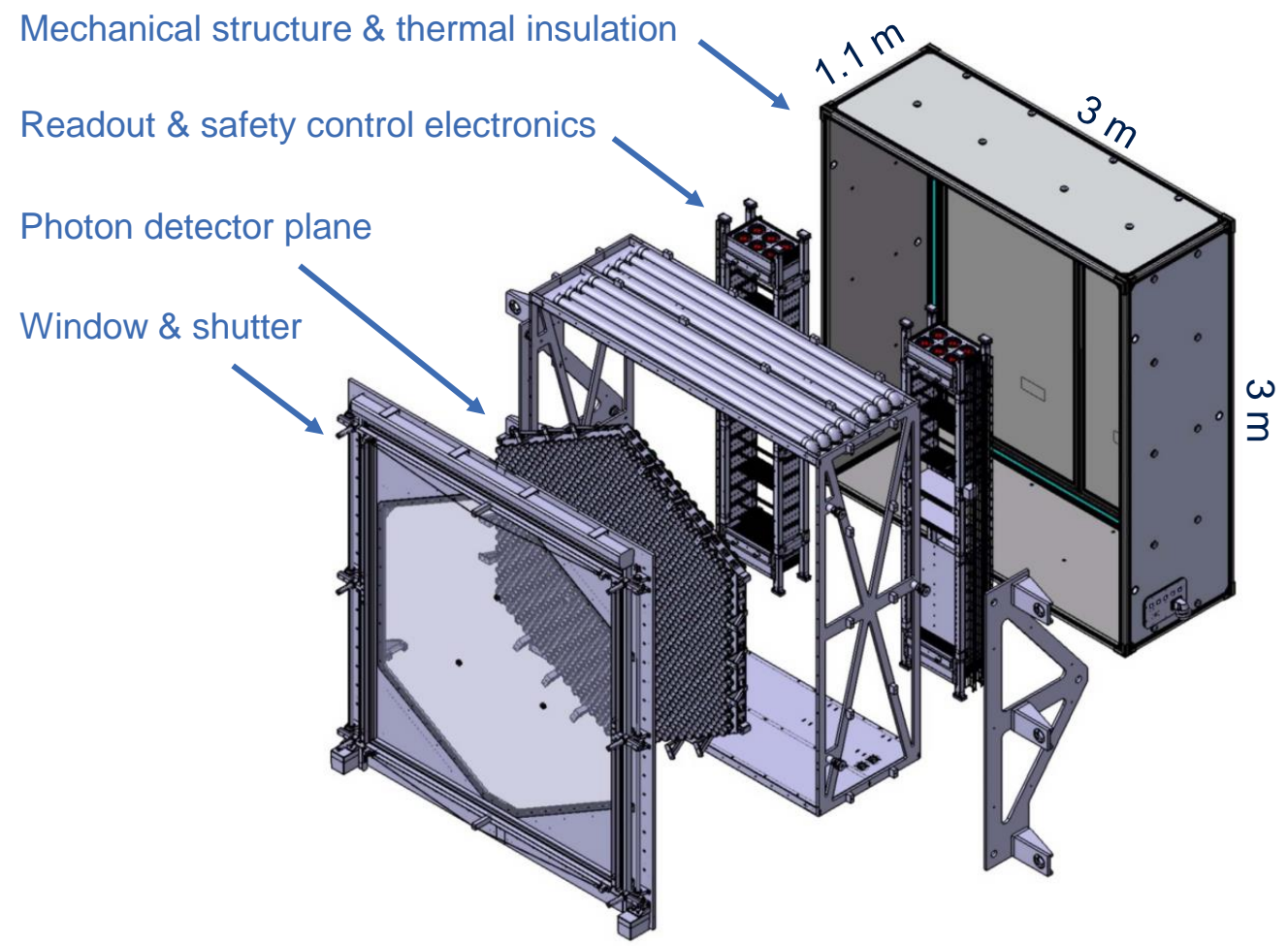

Figure 4. Exploded view of the FlashCam structural design. The readout electronics arranged in crates as well as the photon detector plane units can be seen in this view. 
Figure 4 shows a schematic view of a FlashCam camera, hosting 1758 pixels. Photon detector modules (each with 12 PMTs including pre-amplification circuits, HV supply, and control/monitoring) are physically detached from the readout front-end electronics. The readout electronics boards (serving 24 channels each), as well as trigger and master distribution boards are organized in crates and racks at the back of the camera. The racks can be accessed for installation and maintenance from behind the camera after opening the rear doors. Photon detector plane modules are installed (and exchanged in case of maintenance repair) from the inside of the camera, without the need to remove the optical front system. Cooling inside the camera is performed by means of dry air flow, the dissipated $<4.5 \mathrm{~kW}$ is brought to ground by the liquid cooling system installed at the telescope tower foundation. The camera housing is slightly over-pressured to avoid dust entering through possible gaps in the enclosure.

Bulk data transfer from the front-end to a dedicated server computer over $1 \mathrm{~km} 4 \times 10$ Gbit fibers has been successfully demonstrated with a dead-time free event rate of $>30 \mathrm{kHz}$. Using a highly efficient ethernet protocol developed for this purpose, event traces of $\sim 2000$ channels have been transmitted with full resolution.

The prototype has been used to repeat the verification of performance parameters such as amplitude and time resolution under realistic night-sky background conditions. ${ }^{10}$ Specifically, two different choices of PMTs, developed by Hamamatsu for CTA needs, have been implemented and extensively tested regarding after-pulsing, demonstrating that all PMTs are within requirements $\left(<2 \times 10^{-4}\right.$ above 4 p.e. $)$. Furthermore, the prototype underwent successfully mechanical stress tests, as well as performance tests under different temperature conditions.

\subsection{NectarCAM}

The NectarCAM is designed around the Nectar analogue pipeline readout chip. Incoming PMT signals are continuously sampled at sampling rate of $0.5-2 \mathrm{GHz}$ (default is $1 \mathrm{GHz}$ ). After an event trigger produced inside the camera trigger logic (which is derived from a separate electronics path combining pixel charges), sampling is stopped and signals are digitized and sent by the front-end electronics to a camera server. In order to provide the full dynamic range $(0.5-2000$ p.e.), each pixel signal is sampled with two channels, a low-gain and a high-gain channel. Efficient triggering and readout components ensure a low dead-time of $<5 \%$ at $7 \mathrm{kHz}$ event rate for a full camera.

Figure 6 shows a schematic view of a NectarCAM camera, hosting 1855 pixels. The PMTs and readout electronics are arranged in modules of 7 pixels each, that are installed (and exchanged in case of maintenance repair) from the front side of the camera. Individual modules have been extensively tested and verified for their performance. The total heat dissipation in the camera is around $7.7 \mathrm{~kW}$. Dry air ow is used inside the camera to avoid any risk of condensation, while the camera cooling is ensured with internal fans combined with the exterior liquid cooling system for heat exchange to the outside. The cooling system has been qualified with a demonstrator system.

At the backside of the PMT/electronics modules, modular trigger electronics boards are connected. Since the trigger path is detached from the sampling path, both an analogue trigger (creating analogue trigger sums) and a digital trigger scheme (adding pixel trigger information after simple pixel digitization) were possible. Extensive tests of both options

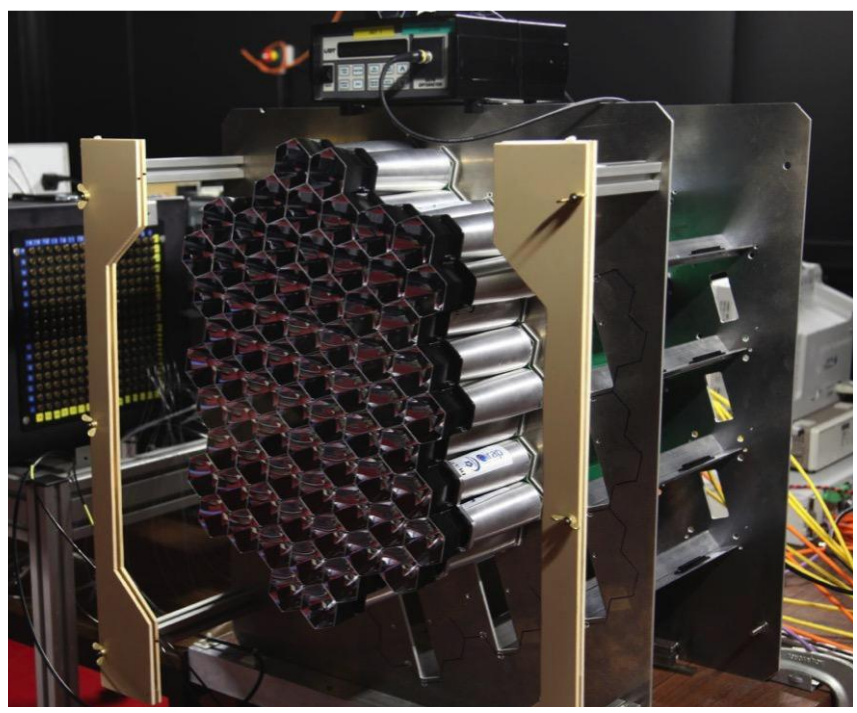

Figure 5. NectarCAM 19-module prototype setup to evaluate the performance of the different trigger logics. were performed, where at the end the digital trigger was chosen. The evaluation of NectarCAM performances is obtained with a 19 module camera setup installed in a dark room at the NectarCAM camera assembly and integration site, as seen in Figure 5. 


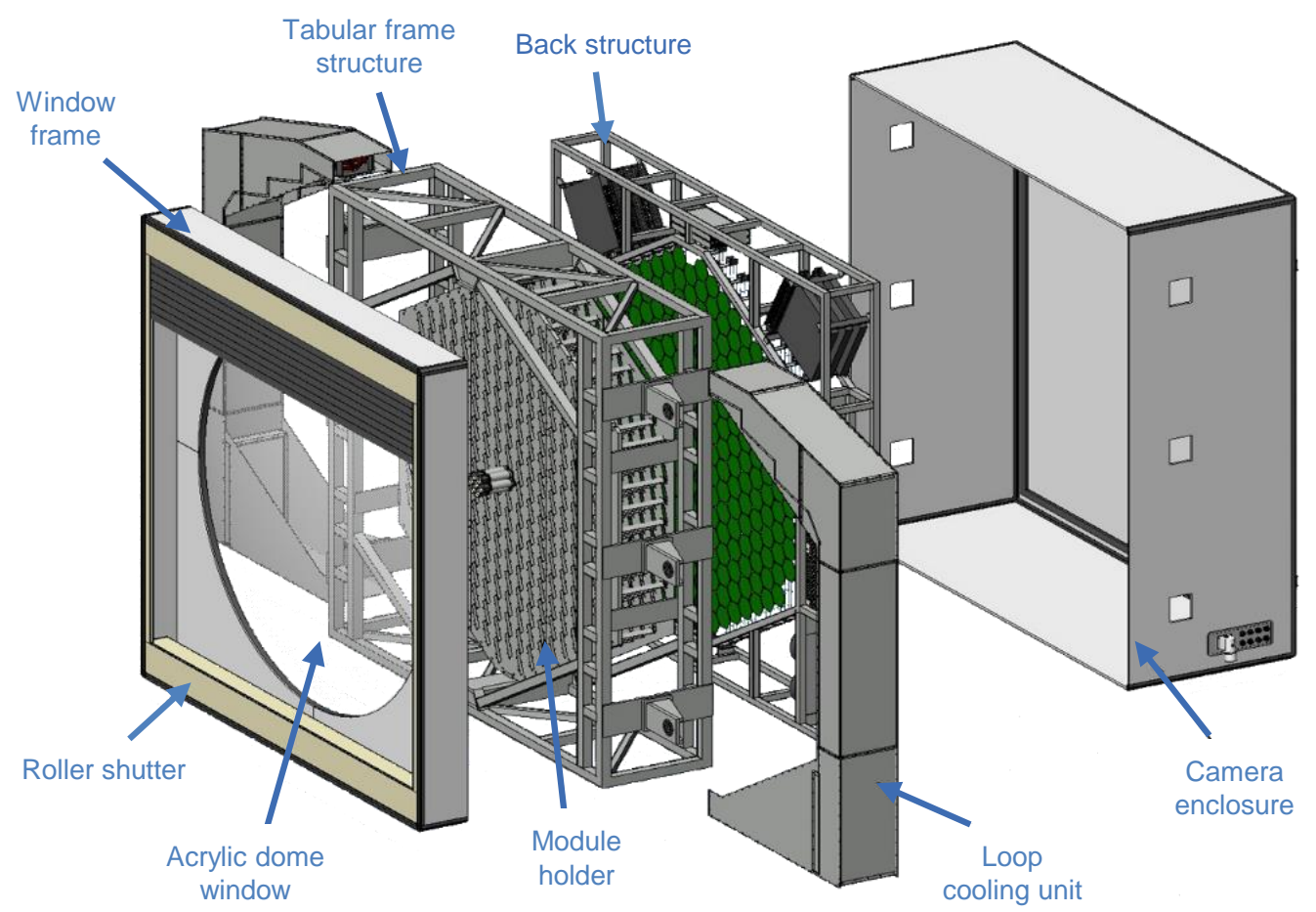

Figure 6. Exploded view of the NectarCAM structural design.

Bulk data transfer from the NectarCAM front-end electronics to a camera server is demanding due to the high pixel data rate, if full event waveforms from all pixels are transmitted for each event. The events are transferred to the camera server through a stack of commercial switches. The full data acquisition has been tested successfully at the nominal $7 \mathrm{kHz}$ rate with the mini-camera. ${ }^{11}$ The dead-time of the instrument is dominated by the Nectar chip readout. The camera server builds the events, performs the online-calibration and is able to store several seconds worth of data taken at nominal rate.

\section{OUTLOOK}

The MST structure, FlashCam and NectarCAM teams are currently preparing towards pre-production telescope structures and cameras, to be deployed on the final CTA sites when they become available. Current plans foresee two telescopes with FlashCam cameras at the Southern site to be ready in mid 2020, and one telescope equipped with a NectarCAM camera at the Northern site on similar timescales.

\section{ACKNOWLEDGMENTS}

The author thanks J.-F. Glicenstein and G. Hermann from the NectarCAM and FlashCam projects for providing material for the presentation and for reviewing the manuscript. We gratefully acknowledge financial support from the agencies and organizations listed here: http://www.cta-observatory.org/consortium_acknowledgments

\section{REFERENCES}

[1] Wild, W., "CTA - building the World's largest ground-based gamma-ray observatory," SPIE (2018).

[2] Cameron, R. A., "Development of a mid-sized Schwarzschild-Couder Telescope for the Cherenkov Telescope Array," SPIE (2012).

[3] Garczarczyk, M., Schlenstedt, S., Oakes, L., and Schwanke, U., "Status of the medium-sized telescope for the cherenkov telescope array," (arXiv:1509.01361), ICRC (2015).

[4] Tiziani, D., Garczarczyk, M., Oakes, L., Schwanke, U., and van Eldik, C., "A pointing solution for the medium size telescopes for the cherenkov telescope array," ICRC (September 2017). 
[5] Melkumyan, D., Oya, I., Bobnar, J., Sah, S., Anutarawiramkul, R., Füssling, M., Sadeh, I., Sekoranja, M., Schmidt, T., Schwanke, U., Sprengler, G., Schwarz, J., and Wegner, P., "Prototyping the central control system for the cherenkov telescope array," SPIE (2018).

[6] Oya, I., Füssling, M., Hinton, J., Mitchell, A., Tositi, G., and Dazzi, F., "Deriving generic telescope use cases for the cherenkov telescope array," SPIE (2018).

[7] Murach, T., Zagar, A., Leben, U., Oya, I., Füssling, M., Dezman, D., Conforti, V., Krack, F., Layard, E., Melkumyan, D., Mosshammer, K., Sadeh, I., Schmidt, T., Schwanke, U., Schwarza, J., and Wiesand, S., "Software testing for the cta observation execution system," SPIE (2018).

[8] Heller, M., "Commissioning of the SST-1M telescope for the Cherenkov Telescope Array," SPIE (2018).

[9] Murach, T., Schwanke, U., Ehlert, D., Garczarczyk, M., Holch, T. L., Kaphle, S., Melkumyan, D., Oakes, L., and Sprengler, G., "Automatic mirror alignment for the medium sized telescopes of the cherenkov telescope array using the bokeh method," SPIE (2018).

[10] Werner, F. and at all., "Performance verification of the FlashCam prototype camera for the Cherenkov Telescope Array," NIM A 876, 31-34 (December 2017).

[11] Tavernier, T., "NectarCAM, a camera for Medium Sized Telescopes of CTA : Status and results," SPIE (2018). 